\title{
Voltammetric and impedimetric behaviour of phytosynthesized nickel nanoparticles
}

\author{
Solomon A. Mamuru ${ }^{1}$ Nurudeen Jaji ${ }^{1,2}$
}

Received: 6 April 2015/Accepted: 1 July 2015/Published online: 18 July 2015

(C) The Author(s) 2015. This article is published with open access at Springerlink.com

\begin{abstract}
The biosynthesis of nickel nanoparticles from nickel chloride using Moringa oleifera leaf extract as reducing agent was successfully carried out; the formation of nickel nanoparticles was confirmed by the use of UVvisible spectroscopy, FTIR spectroscopy, atomic force microscopy, cyclic voltammetry and electrochemical impedance spectroscopy techniques. Optical property showed a colour change from faint light blue of the nickel chloride to dark reddish brown of the nickel nanoparticle after addition of the plant extract, while FTIR confirmed the possible biomolecule responsible for the reduction as anthraquinones; the UV-visible spectroscopy showed the wavelength of nickel nanoparticles at $297 \mathrm{~nm}$, and atomic force microscopy gave images of the nickel nanoparticles as aggregate nanoclusters. Voltammetric and impedimetric behaviour of the nickel nanoparticles towards a one-electron-transfer redox probe was interrogated using cyclic voltammetry and impedance spectroscopy. The equivalent electrical circuit used to fit the measured impedance data indicates that the nanoparticles exhibited patterns characteristic of superimposed porous layer. Electron transfer rate constant $K_{\mathrm{s}}$ and the apparent heterogeneous electron transfer constant $K_{\text {app }}$ were calculated as $6.18 \times 10^{18}$ and $1.60 \times 10^{-3} \mathrm{~cm} \mathrm{~s}^{-1}$, respectively. Such high value is an indication of how fast the nickel nanoparticle can transfer electron from the nanoparticle to the underlying platinum
\end{abstract}

Solomon A. Mamuru

almanto1@yahoo.com

1 Electrochemical NanoLab, Department of Chemistry, Adamawa State University, PMB 25, Mubi 650001, Nigeria

2 Department of Chemistry, Federal College of Education (Technical), Gombe, Nigeria electrode, implying that the nanoparticle can be a potential candidate in the fabrication of biosensors and in catalysis.

Keywords Biomaterial · Nanostructures · Fourier transform infrared spectroscopy $\cdot$ Electrochemical properties

\section{Introduction}

Metallic nanoparticles are of value because their physicochemical and optoelectronic properties are dependent on their unique size, shape, composition and crystallinity. In recent years metallic nanoparticles synthesized using the physical and chemical methods have attracted much interest due to their potential applications in diverse technological areas. The chemical approach is the popular method for the production of metal nanoparticles. However, some chemical methods cannot avoid the use of toxic chemicals in the synthesis protocol; for instance, hazardous substances such as tetrakis hydroxymethyl phosphonium chloride (THPC) [1], poly- $N$-vinyl pyrrolidone (PVP) [2] and hydroxylamine [3] were used for the synthesis of metal nanoparticles in the traditional wet methods. Other dry methods such as UV-irradiation [4] aerosol [5] and lithography [6] are also not considered environmentally friendly. The presence of these toxic chemicals on the surface of nanoparticles limits their medical applications. Suggestions have been made that biological methods of nanoparticle synthesis using microorganisms [7], enzymes [8], and plant extracts [9] or plants [10] are possibly ecofriendly alternatives to chemical and physical methods, which is why focus has now shifted towards using the biological approach in the synthesis of metal nanoparticles. Several microorganism and plant extracts have been 
studied mainly for gold and silver nanoparticles because of their biomedical applications. A recent study is that of the use of Pterocarpus santalinus leaf extract in the synthesis of silver nanoparticles [11] and the synthesis of gold nanoparticles using Piper betle leaf extract [12]. The number of plants used in the biosynthesis keeps growing by the day as more researchers focus their attention to this new and relatively cheap and fast method of metal nanoparticle synthesis. Metal nanoparticles of iron, cobalt, gold, silver and platinum have been widely studied. However, studies carried out on nickel nanoparticles synthesized using both the physical or chemical methods are relatively few [1315] and even none for the biological method. It is in view of this that a study on the biologically synthesized nickel nanoparticles using Moringa oleifera leaf extract is carried out, with a view to establish its potential application in the areas of catalysis and the development of biosensors.

\section{Results and discussion}

\section{Optical property}

Figure 1 shows the optical property of nickel chloride solution, A, M. oleifera leaf extract, B and nickel nanoparticles, $\mathrm{C}$. The change in colour of the nickel chloride from very faint blue to dark reddish brown after addition of the $M$. oleifera leaf extract indicates the formation of the nanoparticles. An observed change in colour indicating the formation of nanoparticles in synthesis involving biological agents was attributed to the excitation of surface plasmon vibrations in the metal nanoparticles [18-20].

\section{UV-visible spectroscopy analysis}

UV-visible spectroscopy is a powerful technique in ascertaining the formation and stability of metal nanoparticles in aqueous solution. Metallic nanoparticles display characteristic optical absorption spectra in the UV-visible region called surface plasmon resonance (SPR) which is due to the physical absorption of light by metallic nanoparticles, and this leads to a coherent oscillation of the conduction electron. This is a small particle effect since it is absent in individual atoms as well as in their bulk structures [21]. The position of SPR is also dependant on size and shape of the particles.

Figure 2 presents the UV-visible spectra of $M$. oleifera leaf extract (A) and nickel nanoparticles synthesized using M. oleifera (B). The UV-visible spectra of nickel nanoparticles show the characteristic fingerprint of the surface plasmon resonance (SPR) spectra with absorbance at 295-350 nm with peak maxima at $\sim 300 \mathrm{~nm}$, which can be attributed to the formation of nickel nanoparticles. The shape of the plasmon band was almost symmetrical, suggesting that the nanoparticles are well dispersed and uniform. Non-uniformity of the nanoparticles leads to a broad absorption peak at higher wavelength and the splitting of a plasmon band into two bands [22, 23]. Also, the shift to lower wavelength of the spectrum indicates that the size of the nickel nanoparticle is small since optical absorption spectrum shifts to lower wavelength with decreasing particle size. $300-400 \mathrm{~nm}$ is the reported UV-visible spectrum of nickel nanoparticle [24]. Multiple peaks observed for the M. oleifera leaf extract may be due to the phytochemicals present in the extract.

\section{Fourier transform infrared spectroscopy analysis}

FTIR spectroscopy analysis was performed to identify the possible biomolecules responsible for the reduction of $\mathrm{Ni}^{2+}$ ions and capping of the reduced nickel nanoparticles synthesized using $M$. oleifera leaf extract. Figure 3 shows the results obtained for $M$. oleifera and nickel nanoparticles.

The results obtained for $M$. oleifera leaf gave IR bands at 3287, 2918, 2850, 1636, 1546, 1401, 1236 and $1047 \mathrm{~cm}^{-1}$. The IR bands for nickel nanoparticles were seen at the following bands: 3238, 2917, 2849, 1555, 1376, 1267,1023 and $910 \mathrm{~cm}^{-1}$. From the bands obtained for $M$. oleifera leaves and nickel nanoparticles, the band at $1636 \mathrm{~cm}^{-1}$ is the only one in $M$. oleifera that is absent in the nickel nanoparticles. This band has been identified as
Fig. 1 Optical images of $0.001 \mathrm{M}$ nickel chloride solution (a), Moringa oleifera leaf extract (b) and nickel nanoparticles synthesized using M. oleifera $(\mathbf{c})$
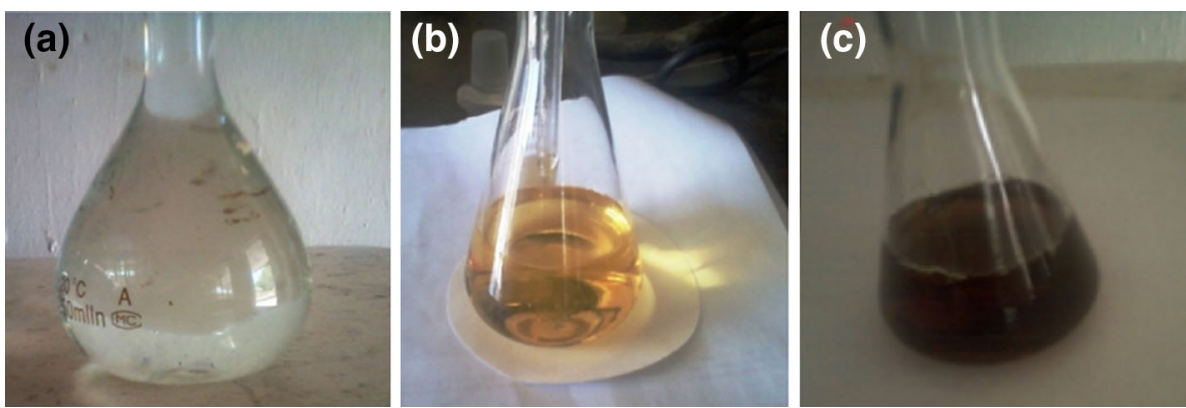


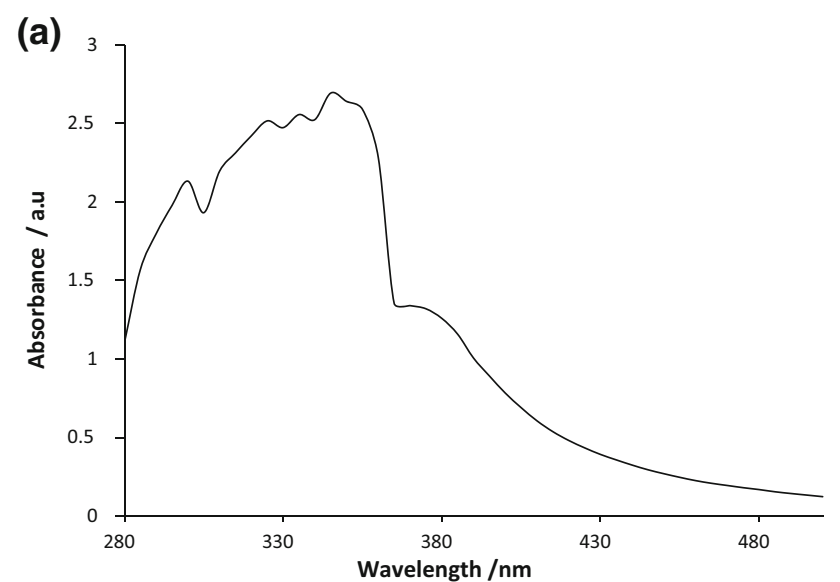

(b)

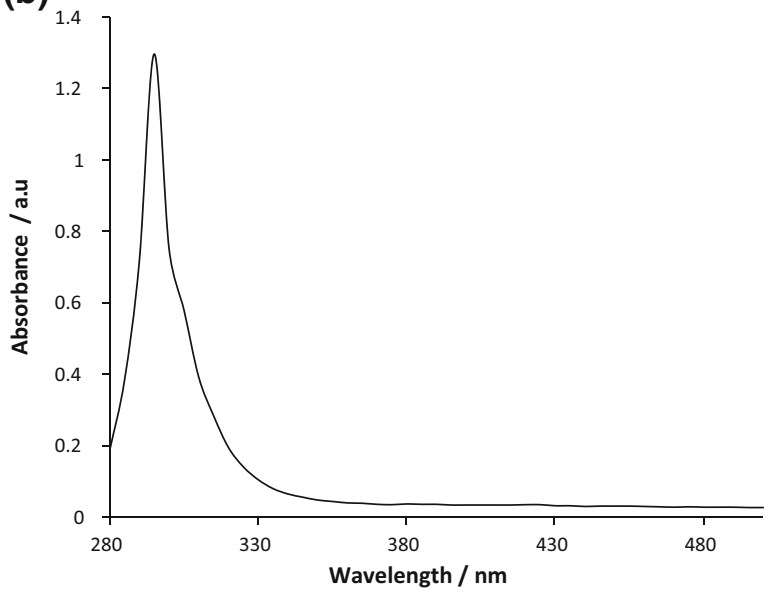

Fig. 2 UV-visible spectra of Moringa oleifera leaf extract (a) and nickel nanoparticles synthesized using $M$. oleifera (b)

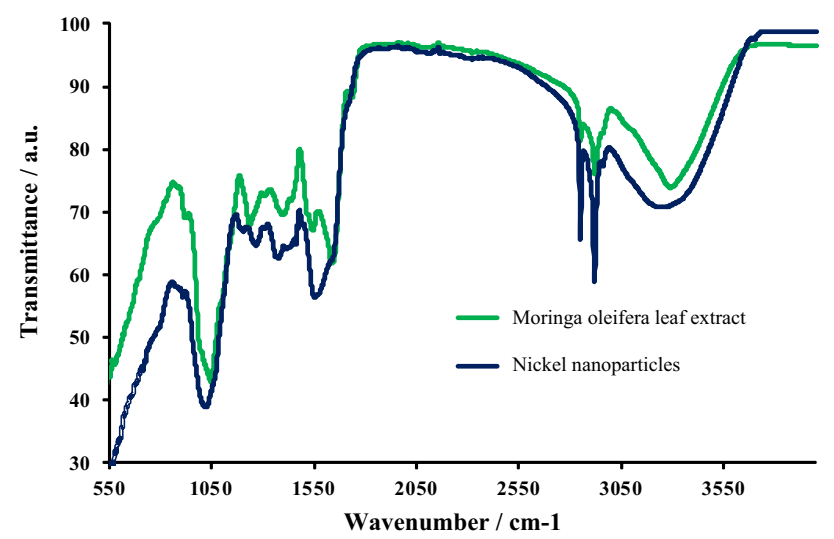

Fig. 3 FTIR spectra of nickel nanoparticles synthesized using Moringa oleifera leaf extract and M. oleifera

belonging to amino aryl ketones. The observed moderate band intensity is as a result of intramolecular hydrogen bonding, other substituents and steric hindrance [25]. Amino aryl ketones show bands at around $1655-1635 \mathrm{~cm}^{-1}$. The band at $1636 \mathrm{~cm}^{-1}$ seen in Fig. 3 is within this range. Also, anthraquinones have been identified as one of the main biochemical components of M. oleifera leaf [26]. Since amino aryl ketones are associated with anthraquinones, it is, therefore, possible that the biomolecule responsible for the reduction of $\mathrm{Ni}^{2+}$ to the nickel nanoparticle is the group of anthraquinones present in the M. oleifera. Test was carried out to confirm the presence of anthraquinone: A cherish red colour was observed after addition of $25 \%$ ammonia solution to the extract, indicating the presence of anthraquinone [26]. Possible structure and mechanism of the reduction process is proposed as presented in Fig. 4.

\section{Atomic force microscopy}

Figure 5 shows the AFM topographical 3D image of nickel nanoparticles. The particles appear as aggregate nanoclusters. Ramesh [27] had earlier reported the aggregation of nickel nanoparticles; this may be due to strongly intermolecular forces such as van der Waals attraction and $\Pi-$ $\Pi$ interaction. For magnetic nanoparticles such as nickel, magnetic dipole-dipole interaction makes this attraction stronger [28].

\section{Electrochemical characterization}

\section{Cyclic voltammetric studies}

Figure 6 compares typical cyclic voltammetric evolutions of bare Pt and Pt/NiNP recorded in $0.005 \mathrm{M}\left[\mathrm{Fe}\left(\mathrm{CN}_{6}\right)\right]^{3-}$ / $\left[\mathrm{Fe}\left(\mathrm{CN}_{6}\right)\right]^{4-}$ solution containing $2.0 \mathrm{M} \mathrm{NaOH}$. It should be noted that $\left[\mathrm{Fe}(\mathrm{CN})_{6}\right]^{3-} /\left[\mathrm{Fe}(\mathrm{CN})_{6}\right]^{4-}$ is an important redox probe known to exhibit one-electron reversible process. The choice of this redox probe in this work is to answer the question as to what extent can the modifying species permit the electron transfer of the $\left[\mathrm{Fe}(\mathrm{CN})_{6}\right]^{3-} /\left[\mathrm{Fe}(\mathrm{CN})_{6}\right]^{4-}$ species to the underlying $\mathrm{Pt}$ electrode. In Fig. 6, the bare $\mathrm{Pt}$ shows a pair of oxidation $\left(E_{\mathrm{ox}}\right)$ and reduction $\left(E_{\mathrm{red}}\right)$ peak potentials at 319 and $181 \mathrm{mV}$ ascribed to $\mathrm{Fe}^{3+} / \mathrm{Fe}^{2+}$. The $\mathrm{Pt} / \mathrm{NiNP}$ modified electrode shows two pair of oxidation and reduction peaks at 329 and $176 \mathrm{mV}$ which is ascribed to $\mathrm{Fe}^{3+} / \mathrm{Fe}^{2+}$ and 580 and $420 \mathrm{mV}$ which is ascribed to $\mathrm{Ni}^{3+} / \mathrm{Ni}^{2+}$ [29]. The presence of the nickel redox process is an indication of the electroactive nature of the nickel nanoparticle. Both electrodes gave formal potential $\left(E_{1 / 2}=E_{\mathrm{ox}}+E_{\mathrm{red}} / 2\right)$ of $250 \mathrm{mV}$ which is the standard electrode potential of $\left[\mathrm{Fe}(\mathrm{CN})_{6}\right]^{3-} /\left[\mathrm{Fe}(\mathrm{CN})_{6}\right]^{4-}$ redox couple.

Next, the type of the diffusion process that could be occurring at the Pt/NiNP electrode was interrogated by using the "diffusion domain approximation" theory developed by Davies and Compton [30, 31] which can also be applied to a three-dimensional electrochemically 


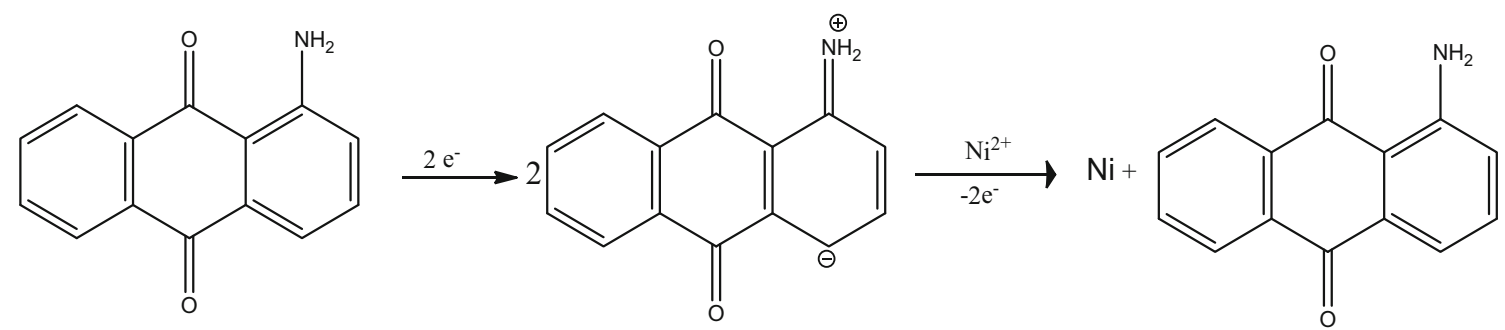

Fig. 4 Proposed structure and mechanism of the reduction process
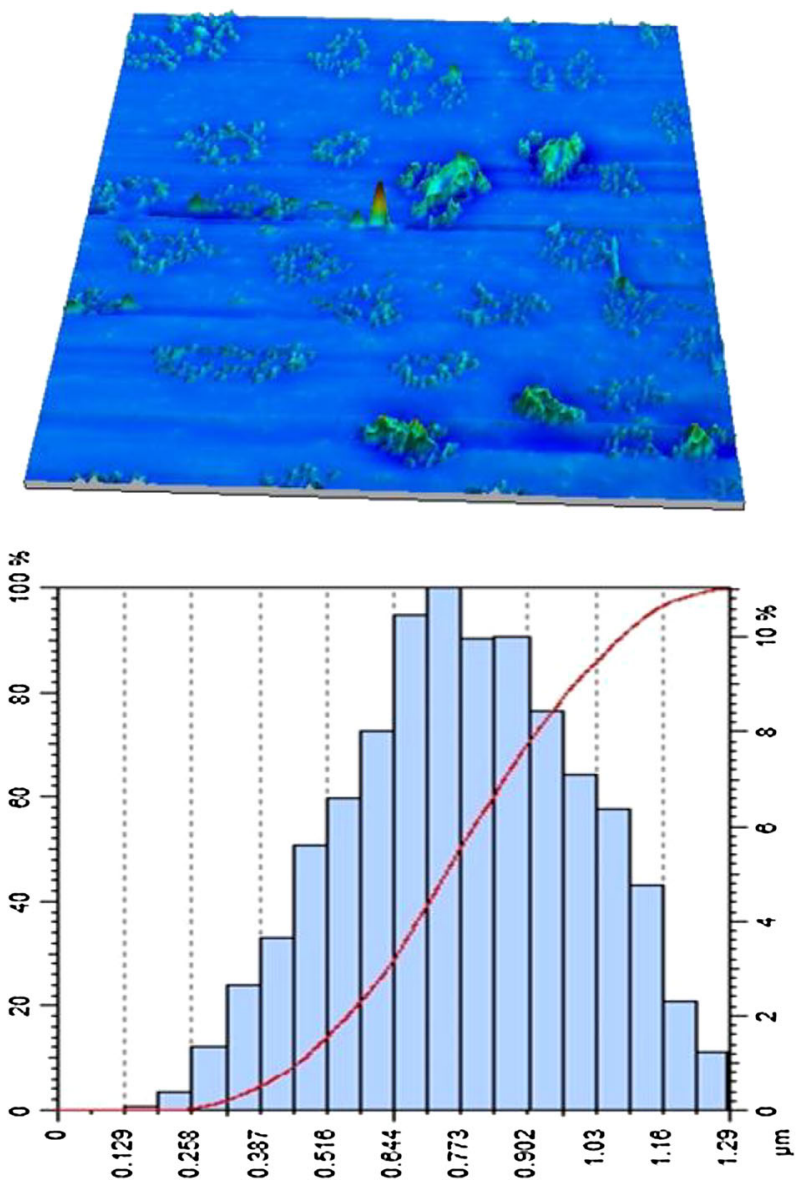

Fig. 5 AFM 3D topographical image and particle sizes distribution of nickel nanoparticles

heterogeneous electrode [32]. It can be seen in Fig. 6 that the voltammetric response by the $\mathrm{Pt} / \mathrm{NiNP}$ electrode is about half the current of the bare Pt electrode. This suggests that the size of the electroactive zones was "micro", but separated with sufficiently large inert blocking material such that the electrode as a whole behaves as a collection of isolated microelectrodes, each of which experiences a convergent diffusion where there is a partial depletion in the concentration of the redox couple to the surface of the $\mathrm{Pt} / \mathrm{NiNP}$ electrode, and hence there was a reduced current response.

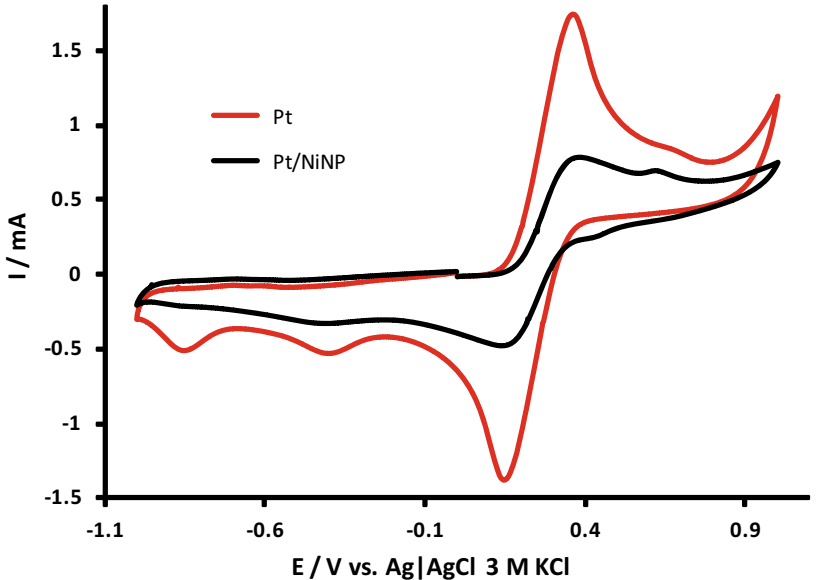

Fig. 6 Comparative cyclic voltammograms of the electrodes in $0.005 \mathrm{M}\left[\mathrm{Fe}(\mathrm{CN})_{6}\right]^{3-} /\left[\mathrm{Fe}(\mathrm{CN})_{6}\right]^{4-} 0.2 \mathrm{M} \mathrm{NaOH}$ solution. Scan rate $100 \mathrm{mVs}^{-1}$

\section{Electrochemical impedance spectroscopy (EIS)}

EIS is a complementary technique to $\mathrm{CV}$; while the latter represents only part of an electrochemical system, the results of the EIS measurements give a fuller description of the electrochemical system, [33,34] providing vital information about processes occurring at the electrode/electrolyte interface.

Figure 7a, c presents the Nyquist plots for the electrodes studied in $2.0 \mathrm{M} \mathrm{NaOH}$ solution containing $0.005 \mathrm{M}$ $\left[\mathrm{Fe}(\mathrm{CN})_{6}\right]^{3-} /\left[\mathrm{Fe}(\mathrm{CN})_{6}\right]^{4-}$. The impedance spectra of the electrodes were satisfactorily fitted with the proposed equivalent electrical circuits (Fig. 8). The bare Pt electrode was fitted with circuit (a), while Pt/NiNP electrode was fitted with circuit (b). The fitting parameters involve the electrolyte resistance $\left(R_{\mathrm{S}}\right)$, electron transfer resistance $\left(R_{\mathrm{ct}}\right)$ and constant phase element (CPE). The apparent heterogeneous electron transfer rate constant $\left(k_{\text {app }}\right)$ values of the electrodes were obtained using Eq. (1).

$k_{\mathrm{app}}=\frac{R T}{n^{2} F^{2} A R_{\mathrm{ct}} c}$

where $n$ is the number of electron transferred, $A$ is the area of the electrode, $c$ is the concentration of the $\left[\mathrm{Fe}(\mathrm{CN})_{6}\right]^{3-}$ 
(a)

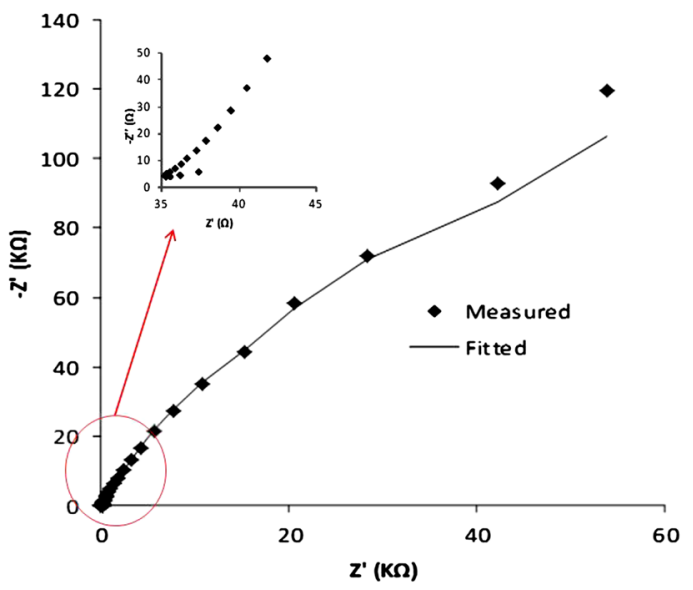

(b)

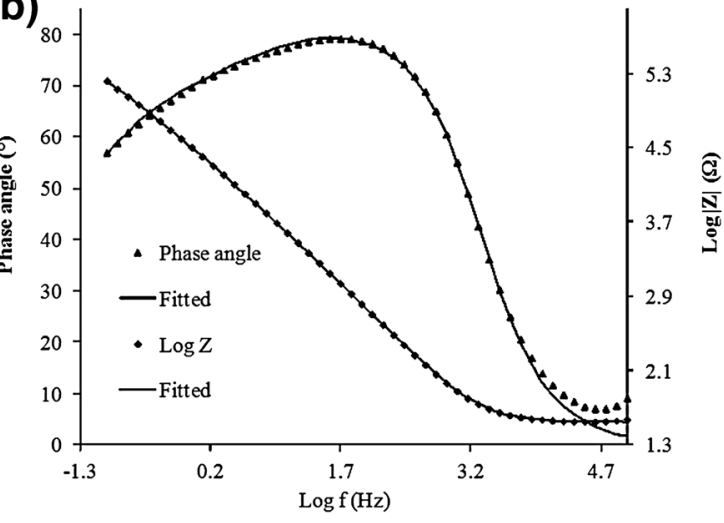

(c)
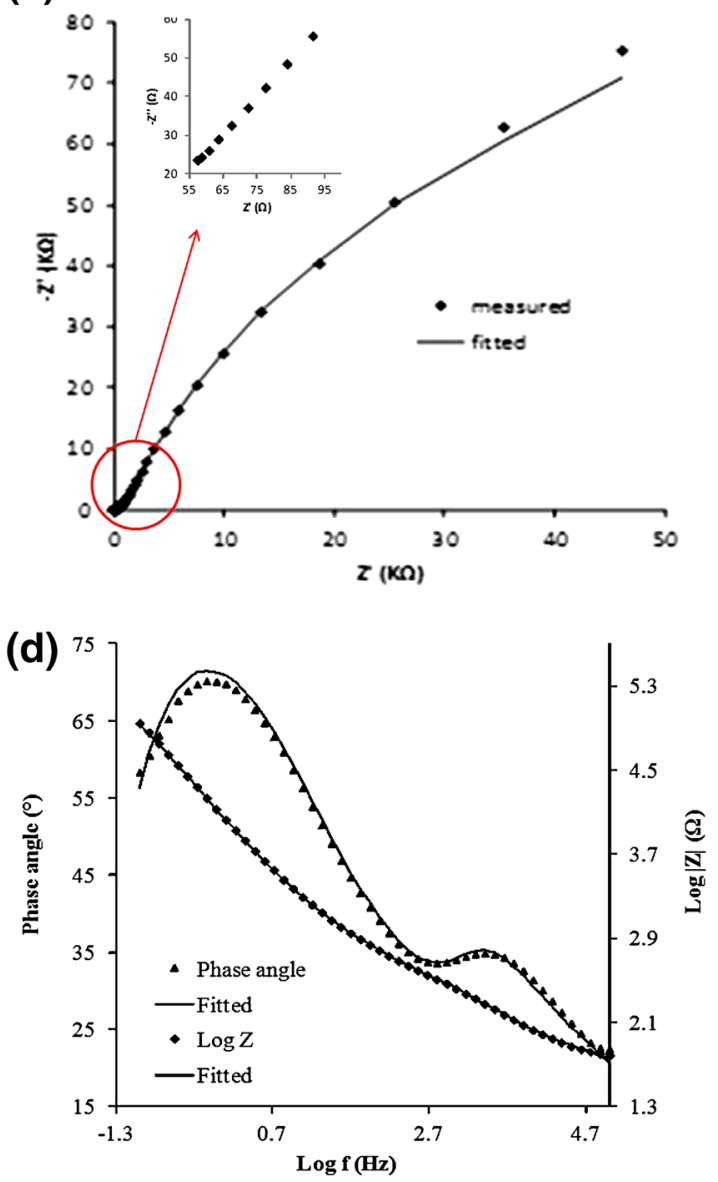

Fig. 7 Nyquist and bode plots of bare platinum a, b and Pt/NiNP c, d electrodes in $0.005 \mathrm{M}\left[\mathrm{Fe}(\mathrm{CN})_{6}\right]^{3-} /\left[\mathrm{Fe}(\mathrm{CN})_{6}\right]^{4-} 0.2 \mathrm{M} \mathrm{NaOH}$ solution

Fig. 8 Equivalent electrical circuit

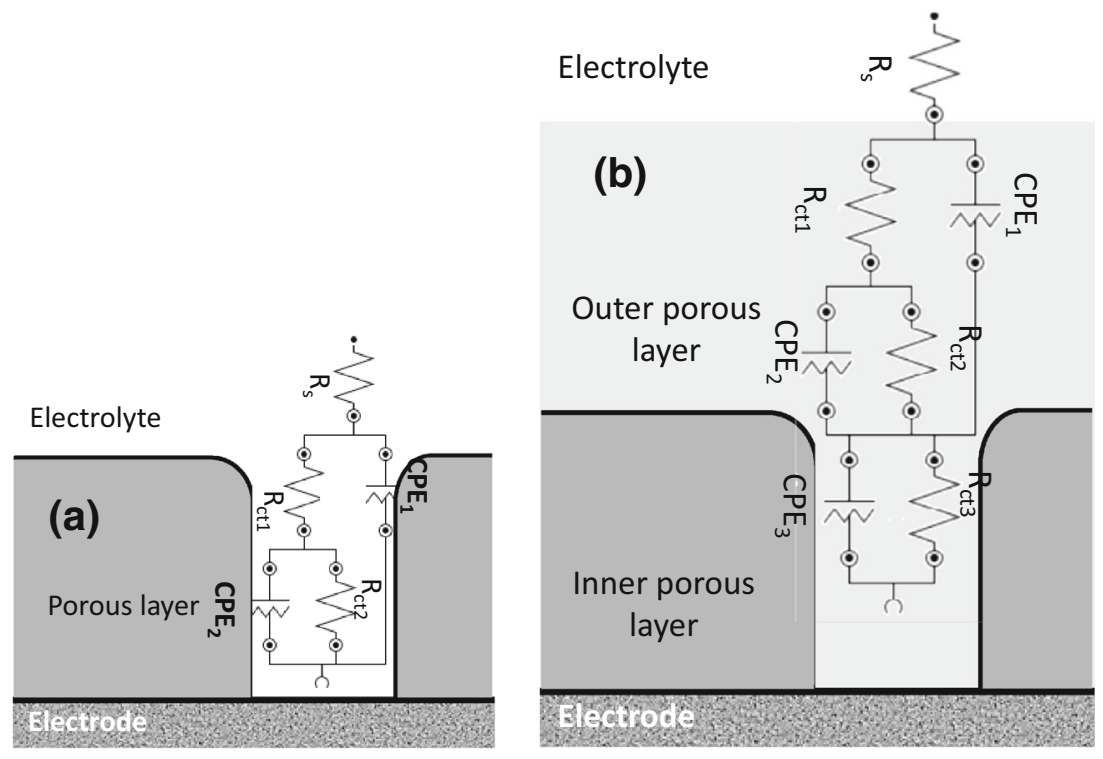

(in $\mathrm{mol} \mathrm{cm}{ }^{-3}$, the concentration of $\left[\mathrm{Fe}(\mathrm{CN})_{6}\right]^{3-}$ and $\left[\mathrm{Fe}(\mathrm{CN})_{6}\right]^{4-}$ are equal), $R$ is the ideal gas constant, $T$ is the absolute temperature $(K)$ and $F$ is the Faraday constant.
The calculated values are shown in Table 1 , wherein the $k_{\text {app }}$ values decreased as: Pt/NiNP $>$ Pt electrode, indicating that electron transfer processes between the redox 
Table 1 Summary of the measured electrochemical parameters for the electrodes studied

\begin{tabular}{lrllll}
\hline Electrodes & $R_{\mathrm{ct}} / \Omega$ & $K_{\text {app }}\left(\times 10^{3}\right) \mathrm{cm} \mathrm{s}^{-1}$ & $K_{\mathrm{s}} / \mathrm{cm} \mathrm{s}^{-1}$ & $\Delta E / V$ & $E_{1 / 2} / V$ \\
\hline Platinum & 37,870 & 0.67 & $8.82 \times 10^{5}$ & 0.138 & 0.25 \\
Nickel nanoparticle & 2759 & 1.60 & $6.18 \times 10^{18}$ & 0.153 & 0.25 \\
\hline
\end{tabular}

probe and the underlying Pt surface are much easier at the $\mathrm{Pt} / \mathrm{NiNP}$ electrode than at the Pt electrode. The impedance of the CPE $\left(Z_{\mathrm{CPE}}\right)$ is defined as in (Eq. 2).

$$
Z_{\mathrm{CPE}}=\left[Q(j \omega)^{n}\right]^{-1}
$$

where $\mathrm{Q}$ is the frequency-independent constant related to the interface, $j=\sqrt{-1}, \omega$ is the radial frequency, the exponent $\mathrm{n}$ arises from the slope of $\log Z$ versus $\log f$ (and has values $-1 \leq n \leq 1)$. If $n=0$, the CPE behaves as a pure resistor; $n=1, \mathrm{CPE}$ behaves as a pure capacitor; $n=-1$ CPE behaves as an inductor; while $n=0.5$ corresponds to Warburg impedance (W) which is associated with the domain of mass transport control arising from the diffusion of ions. In short, CPE arises from several factors such as (1) the nature of the electrode (e.g. roughness and polycrystallinity), (2) distribution of the relaxation times due to heterogeneities existing at the electrode/electrolyte interface, (3) porosity and (4) dynamic disorder associated with diffusion. In the circuits, notice that the Warburg in a typical Randles equivalent electrical circuit has been replaced by the $\mathrm{CPE}_{2}$, which simply describes the porous nature of the concerned electrode. In fact, the equivalent electrical circuit used to fit the Pt/NiNP electrode exhibited pattern characteristic of a superimposed porous layer electrode [35]. From the Bode plots (Fig. 7b, d), the slopes of the $\log Z$ versus $\log f$ plot at the mid-frequency region are less than the ideal -1.0 for pure capacitive behaviour, which is indicative of pseudo-capacitive behaviour. The data from the Bode plots (i.e. phase angle vs. $\log f$ ) further confirm the absence of typical capacitive behaviour as the observed phase angles are less than the $90^{\circ}$.

\section{Scan rate studies}

The effect of scan rate on the peak current of Pt and Pt/ NiNP modified electrode was examined. With the increase in scan rates from 100 to $800 \mathrm{mVs}^{-1}$, the currents for both peaks increased as shown in Figs. 9a and 10a. The anodic and cathodic peak currents are linearly proportional to the square root of the scan rate which is expected for a diffusion controlled electrode process, Figs. $9 \mathrm{~b}$ and 10b. At higher scan rates, the peak currents were proportional to the scan rate indicative of a surface confined redox process. According to the method demonstrated by Laviron [36], the charge transfer coefficient, $\alpha$, and the apparent heterogeneous charge transfer rate constant, $k_{\mathrm{s}}$, of a surface-confined redox couple can be estimated in cyclic voltammetry from the variation of the $E_{\mathrm{ox}}$ and $E_{\mathrm{red}}$ peak potentials as a function of the logarithm of scan rates. For higher scan rates, this theory predicts a linear dependence of $E_{\mathrm{p}}$ versus $\log v$, which can be used to extract the kinetic parameters of $\alpha$ and $k_{\mathrm{s}}$ from the slope and intercept of such plots, respectively. Figures $9 \mathrm{c}$ and $10 \mathrm{c}$ show the variation of the peak potentials versus the logarithm of the scan rate obtained from the cyclic voltammograms of the electrodes.

It is found that the $\Delta E_{\mathrm{p}}$ values are proportional to the logarithm of the scan rate, for higher scan rates. A plot of $E_{\mathrm{p}}$ versus $\log v$ yields two straight lines with slopes of $2.3 \mathrm{RT} /(1-\alpha) n F$ for anodic and $-2.3 \mathrm{RT} / \alpha n F$ for the cathodic peak. The value of $\alpha$ was calculated to be 0.64 for Pt and 0.39 for the Pt/NiNP. Laviron's equation can also be used to determine the electron transfer rate $\left(k_{\mathrm{s}}\right)$ between the NiNP and the underlying electrode, and from the value of $\Delta E_{\mathrm{p}}$ corresponding to different scan rates, an estimated value of $k_{\mathrm{s}}$ was calculated to be $6.18 \times 10^{18} \mathrm{~cm} \mathrm{~s}^{-1}$ for $\mathrm{NiNP}$ and $8.82 \times 10^{5} \mathrm{~cm} \mathrm{~s}^{-1}$ for bare platinum. This result closely agrees with the $k_{\text {app }}$ trend from the EIS data. The high value of electron transfer rate constant is an indication of the ability of the nickel nanoparticle to promote electron transfer between it and the bare electrode surface.

\section{Conclusions}

The phytosynthesis of nickel nanoparticles from nickel chloride using $M$. oleifera leaf extract as reducing agent was successfully carried out; the use of advanced spectroscopic, microscopic and electrochemical techniques confirms the formation of nickel nanoparticles. Optical property showed a colour change from faint light blue of the nickel chloride to dark reddish brown of the nickel nanoparticle after addition of the plant extract. FTIR confirmed the possible biomolecule responsible for the reduction as group of amino aryl ketones; the UV-visible spectroscopy showed the wavelength of nickel nanoparticles at $297 \mathrm{~nm}$, the wavelength having shifted to lower values expected of nanoparticle surface plasmon resonance. Atomic force microscopy gave images of the nickel nanoparticles as aggregate nanoclusters having particle size diameter of $\sim 70 \mathrm{~nm}$. Voltammetric and impedimetric behaviour of the nickel nanoparticles towards a one-electron-transfer redox probe was investigated using cyclic voltammetry and impedance spectroscopy. The equivalent 


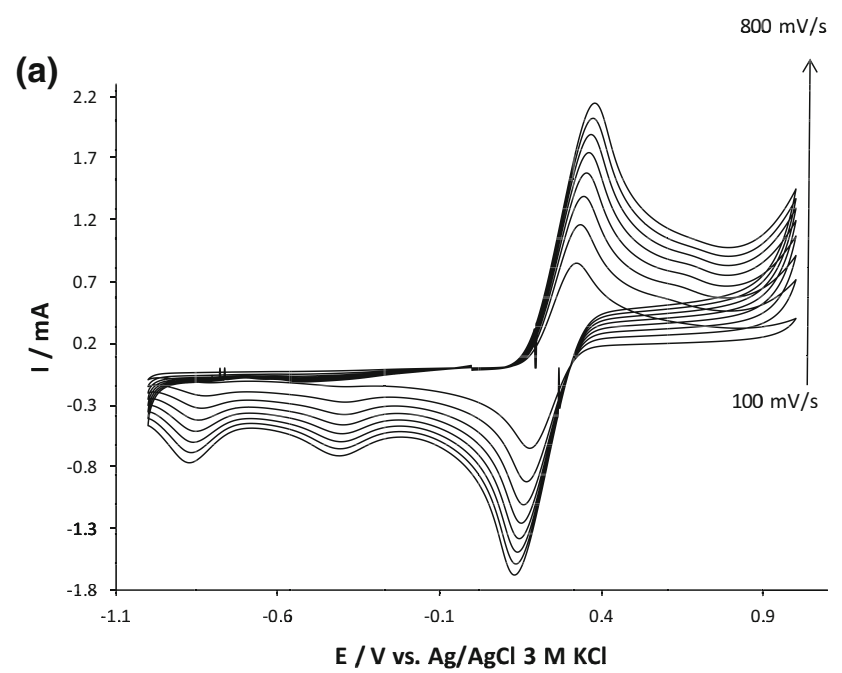

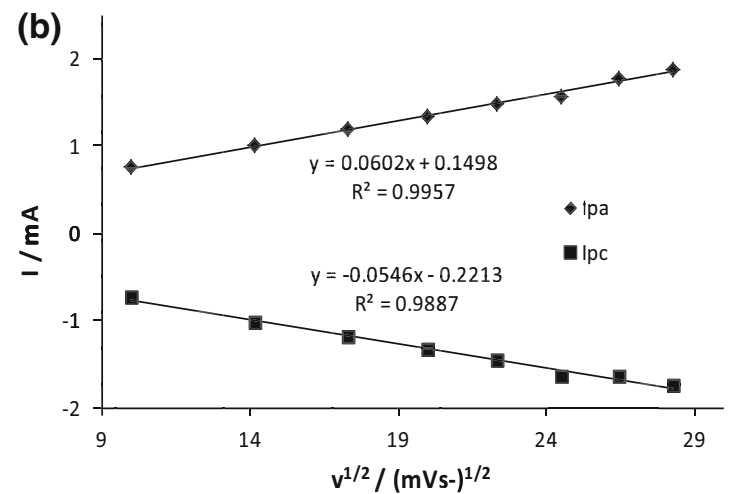

Fig. 9 Cyclic voltammograms of the Pt electrode at different scan rates in $0.005 \mathrm{M}\left[\mathrm{Fe}(\mathrm{CN})_{6}\right]^{3-} /\left[\mathrm{Fe}(\mathrm{CN})_{6}\right]^{4-} 0.2 \mathrm{M} \mathrm{NaOH}$ solution $(\mathbf{a})$, scan rates from 100 to $800 \mathrm{mV} / \mathrm{s}$. Dependence of peak current $\left(I_{\mathrm{pa}}\right.$ and

electrical circuit used to fit the measured impedance data of the Pt/NiNP electrode indicates that the nanoparticles exhibited patterns characteristic of superimposed porous layer. Electron transfer rate constant $\mathrm{k}_{\mathrm{s}}$ and the apparent heterogeneous electron transfer constant $k_{\text {app }}$ were calculated as $6.18 \times 10^{8}$ and $1.60 \times 10^{-3} \mathrm{~cm} \mathrm{~s}^{-1}$, respectively. Such high value is an indication of how fast the nickel nanoparticle can transfer an electron from the nanoparticle to the underlying platinum electrode, implying that the nanoparticle can be a potential candidate as a biosensor and in catalysis.

\section{Experimental}

\section{Materials and reagents}

Fresh leaves of M. oleifera (Drumstick tree) were obtained locally from Adamawa State University campus, Mubi, Adamawa State, Nigeria. All chemicals and equipment

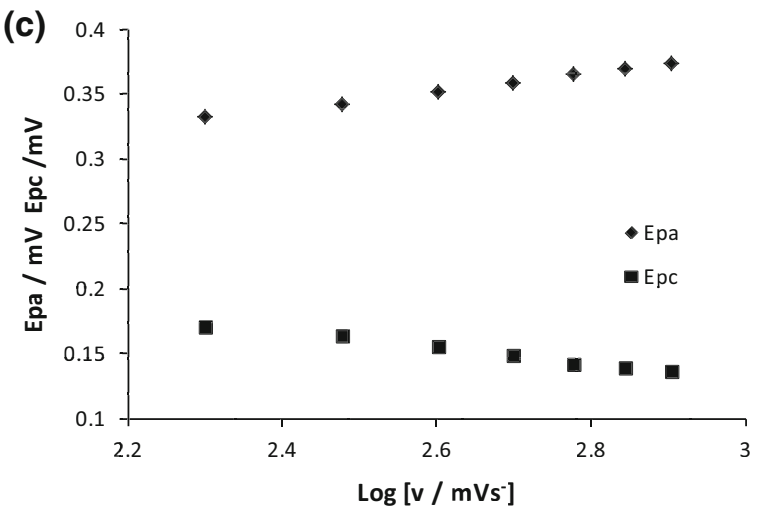

$\left.I_{\mathrm{pc}}\right)$ versus square root the scan rate $\left(v^{1 / 2}\right)(\mathbf{b})$ and plot of the variation of peak potential versus logarithm of the scan rates (c)

were obtained from the Chemistry laboratories of Adamawa State University. Ethanol $99.7 \%$ was obtained from Guangdong Guangzhou Chemical Factory Ltd., China, sodium hydroxide 99-100\%, Sigma-Aldrich Laborchemikalien Gmbh, nickel chloride $97 \%$, BDH Chemicals Ltd., Poole, England; potassium ferrocyanide and potassium ferricyanide $97 \%$, Kem Light Laboratories.

\section{Preparation of Moringa oleifera fresh leaves extract}

Adaptation of the synthesis process was from Gopinath et al. [11] and briefly explained. Twenty grams of fresh leaves of M. oleifera was washed thoroughly with distilled water followed by ultra-pure deionized water and then cut into small pieces. The cut pieces placed in a conical flask containing $100 \mathrm{~mL}$ of ultra-pure deionized water. A magnetic bulb was introduced into the mixture and boiled for 10 min using a magnetic stirrer. The mixture was cooled to room temperature and filtered using Whatman filter paper No. 1. 


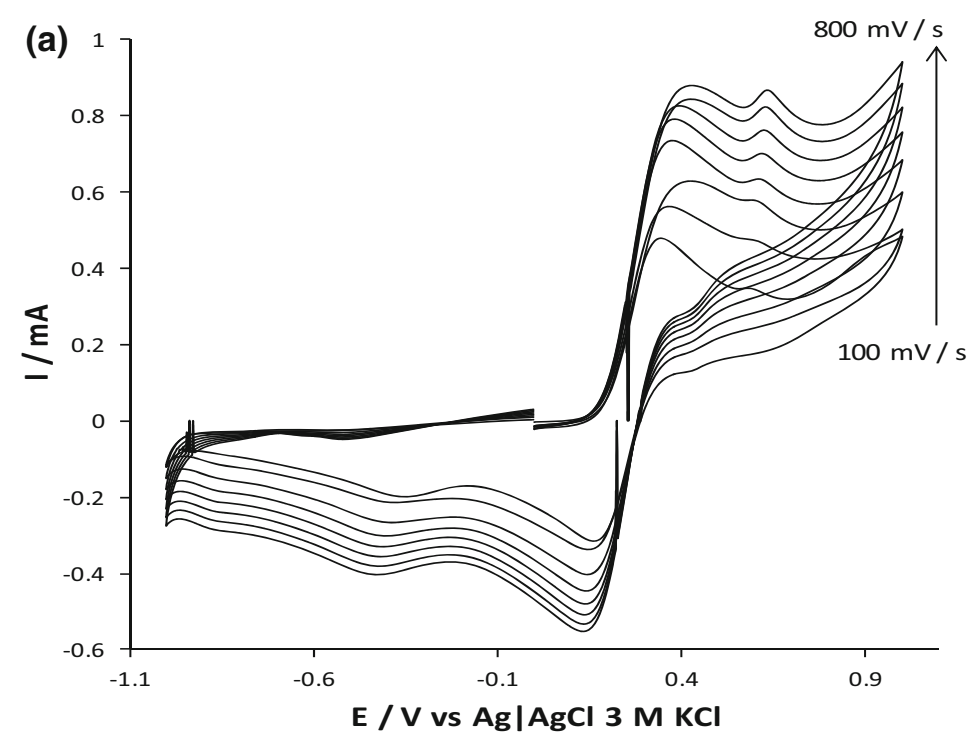

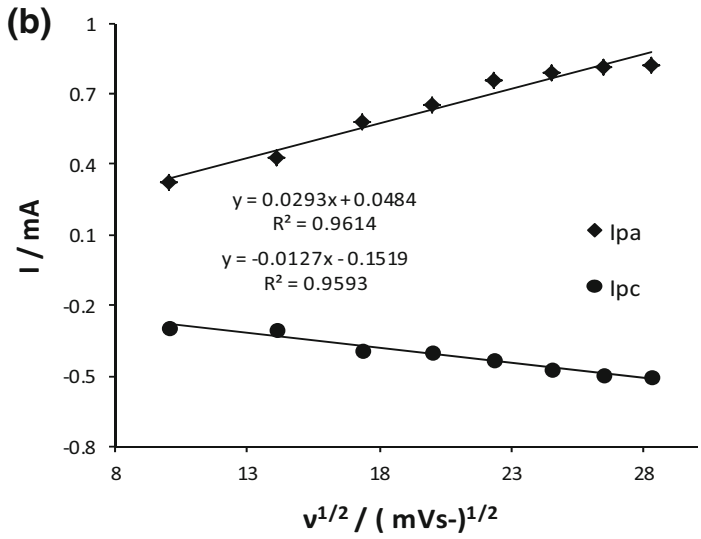

Fig. 10 Cyclic voltammograms of the Pt/NiNP modified electrode at different scan rates in $0.005 \mathrm{M}\left[\mathrm{Fe}(\mathrm{CN})_{6}\right]^{3-} /\left[\mathrm{Fe}(\mathrm{CN})_{6}\right]^{4-} 0.2 \mathrm{M}$ $\mathrm{NaOH}$ solution (a), scan rates from 100 to $800 \mathrm{mV} / \mathrm{s}$. Dependence of

\section{Synthesis of nickel nanoparticles}

A total of $10 \mathrm{~mL}$ of $M$. oleifera leaf extract was added dropwise with continuous stirring for $1 \mathrm{~h}$ to $50 \mathrm{~mL}$ of $0.001 \mathrm{M}$ nickel chloride solution. A colour change was observed, indicating the formation of nickel nanoparticles $[16,17]$. The mixture was centrifuged for $10 \mathrm{~min}$ at $3000 \mathrm{rpm}$. The nanoparticles were washed with ultra-pure deionized water followed with ethanol and allowed to dry at room temperature.

\section{UV-visible and FTIR characterization}

The solution chemistry of the nickel nanoparticles was studied by UV-visible spectrophotometer at wavelength of $300-700 \mathrm{~nm}$ on a Jenway $6405 \mathrm{UV}$-visible spectrophotometer and scan range of $4000-400 \mathrm{~cm}^{-1}$ on

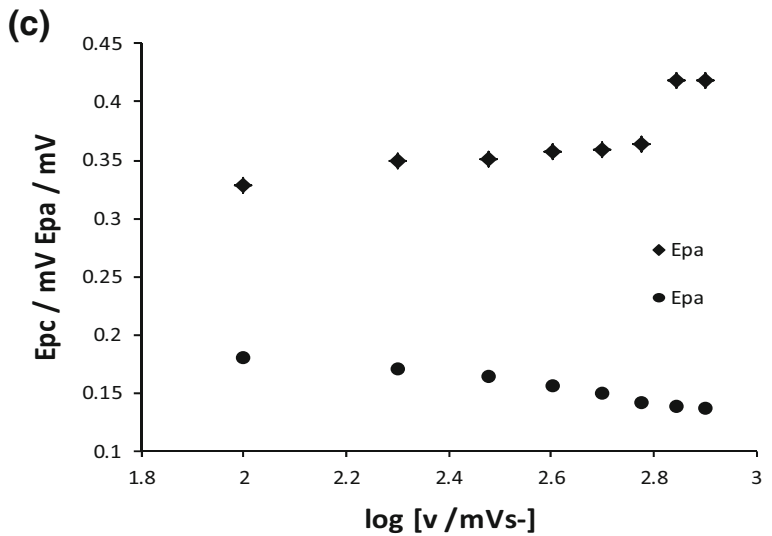

peak current $\left(I_{\mathrm{pa}}\right.$ and $\left.I_{\mathrm{pc}}\right)$ versus square root the scan rate $\left(v^{1 / 2}\right)(\mathbf{b})$ and plot of the variation of peak potential versus logarithm of the scan rates $(\mathbf{c})$

a PerkinElmer Frontier 400 MIR/NIR FTIR spectrophotometer.

\section{Surface characterization}

Topographical images of the nickel nanoparticles were obtained with an Agilent 5500 atomic force microscope employing the contact mode technique with a cantilever having resonant frequency of $17 \mathrm{kHz}$ and spring constant of $0.8 \mathrm{~N} / \mathrm{m}$. Sheet of mica was used as a substrate for placing the nanoparticles.

\section{Electrochemical characterization}

All electrochemical measurements were performed with an Autolab PGSTAT $302 \mathrm{~N}$ driven by NOVA software version 1.8. A three-electrode system was employed for this 
study. The counter electrode was a platinum wire, and an $\mathrm{Ag} \mid \mathrm{AgCl} 3 \mathrm{M} \mathrm{KCl}$ used as a reference electrode.

\section{Electrode preparation}

$10 \mu \mathrm{L}$ of the nickel nanoparticles solution dissolved in carbon tetrachloride $\left(\mathrm{CCl}_{4}\right)$ was placed on the surface of a platinum electrode ( $3 \mathrm{~mm}$ diameter) already polished with alumina slurry (nanopowder) using a micropipette and allowed to dry at room temperature to prepare the platinum-nickel nanoparticles electrode $(\mathrm{Pt} / \mathrm{NiNP})$. The electrochemical properties of the $\mathrm{Pt} / \mathrm{NiNP}$ were studied with an outer sphere redox couple of $0.005 \mathrm{M}$ potassium ferricyanide and potassium ferrocyanide in $0.2 \mathrm{M} \mathrm{NaOH}$ solution using cyclic voltammetry and electrochemical impedance spectroscopy, EIS.

Acknowledgments Adamawa State University and TETFund IBR research grant are acknowledged for providing the resources used in this work. NJ will like to thank Federal College of Education (Technical), Gombe, for postgraduate fellowship.

Authors contribution NJ carried out synthesis and characterization of the nickel nanoparticle, SAM captured the AFM images, read through the manuscript and prepared it for submission. Both authors read through the manuscript before submission.

\section{Compliance with ethical standards}

Conflict of interest The authors have no competing interest.

Open Access This article is distributed under the terms of the Creative Commons Attribution 4.0 International License (http://crea tivecommons.org/licenses/by/4.0/), which permits unrestricted use, distribution, and reproduction in any medium, provided you give appropriate credit to the original author(s) and the source, provide a link to the Creative Commons license, and indicate if changes were made.

\section{References}

1. Porta, F., Prati, L., Rossi, M., Coluccia, S., Martra, G.: Metal sols as a useful tool for heterogeneous gold catalyst preparation: reinvestigation of a liquid phase oxidation. Catal. Today 61, 165-172 (2000)

2. Hoppe, C.E., Lazzari, M., Pardinas-Blanco, I., Lopez-Quintela, M.A.: One-step synthesis of gold and silver hydrosols using poly( $N$-vinyl-2-pyrrolidone) as a reducing agent. Langmuir 22, 7027-7034 (2006)

3. Lyon, J.L., Fleming, D.A., Stone, M.B., Schiffer, P., Williams, M.E.: Synthesis of Fe oxide core/Au shell nanoparticles by iterative hydroxylamine seeding. Nano Lett. 4, 719-723 (2004)

4. Lu, Y., Mei, Y., Schrinner, M., Ballauf, M., Moller, M.W., Breu, J.: In situ formation of Ag nanoparticles in spherical polyacrylic acid brushes by UV-irradiation. J. Phys. Chem. C 111, 7676-7681 (2007)

5. Swihart, M.T.: Vapour-phase synthesis of nanoparticles. Curr. Opin. Colloid Interface. 8, 127-133 (2003)
6. Eustis, S., El-Sayed, M.A.: Why gold nanoparticles are more precious than pretty gold: noble metal surface plasmon resonance and its enhancement of the radiative and nonradiative properties of nanocrystals of different shapes. Chem. Soc. Rev. 35, 209-217 (2006)

7. Joerger, R., Klaus, T., Granqvist, C.G.: Biologically produced Ag-C composite for optically functional thin film coatings. Adv. Mater. 12, 407-409 (2000)

8. Ingle, A., Rai, M., Gade, A., Bawaskar, M.: Fusarium solani: a novel biological agent for the extracellular synthesis of silver nanoparticles. J. Nanoparticle Res. 11, 2079-2085 (2009)

9. Lee, H.J., Lee, G., Jang, N.R., Yun, J.M., Song, J.Y., Kim, B.S.: Biological synthesis of copper nanoparticles using plant extract. Nanotechnology 1, 371-374 (2011)

10. Govindaraju, K., Tamilselvan, S., Kiruthiga, V., Singaravelu, G.: Biogenic silver nanoparticles by Solanum torvum and thier promising antibacterial activity. J. Biopestic. 3, 394-399 (2010)

11. Gopinath, K., Gowri, S., Arumugam, A.: Phytosynthesis of silver nanoparticles using Pterocarpus santalinus leaf extract and their antibacterial properties. J. Nanostruct. Chem. 3, 68 (2013)

12. Gaware, U., Kamble, V., Balaprasad, A.: Ecofriendly synthesis of anisotropic gold nanoparticles: a potential candidate of SERS studies. Int. J. Electrochem. 2012, 6-12 (2012)

13. Adekunle, A.S., Ozoemena, K.I.: Electron transport and electrocatalytic properties of MWCNT/nickel nanocomposites: hydrazine and diethyl aminoethanethiol as analytical probes. J. Electroanal. Chem. 645, 41-49 (2010)

14. Babu, R.S., Prabhu, P., Narayanan, S.S.: Green synthesized nickel nanoparticles modified electrode in ionic liquid medium and its application towards determination of biomolecules. Talanta 110, 135-143 (2013)

15. Salimi, A., Noorbakhash, A., Sharifi, E., Semnani, A.: Highly sensitive sensor for picomolar detection of insulin at physiological $\mathrm{pH}$, using $\mathrm{GC}$ electrode modified with guanine and electrodeposited nickel oxide nanoparticles. Biosens. Bioelectron. 24, 792-798 (2008)

16. Vinod, V.T.P., Saravanan, P., Sreedhar, B., KeerthiDevi, D., Sashidhar, R.: A facile synthesis and characterisation of $\mathrm{Ag}, \mathrm{Au}$ and $\mathrm{Pt}$ nanoparticles using a natural hydrocolloid gum kondagogu (Cochlospermum gossypium). Colloid Surface B 83, 291-298 (2011)

17. MubarakAli, D., Thajuddin, N., Jeganathan, K., Gunasekaran, M.: Plant extract mediated synthesis of silver and gold nanoparticles and its antibacterial activity against clinically isolated pathogens. Colloid Surface B 85, 360-365 (2011)

18. Patil, R.S., Kokate, M.R., Kolekar, S.S.: Bioisnpired synthesis of highly stabilized silver nanoparticles using Ocimum tenuiflorum leaf extract and their antibacterial activity. Spectrochim. Acta A 91, 234-238 (2012)

19. Narayanan, K.B., Sakthivel, N.: Green synthesis of biogenic metal nanoparticles by terrestial and aquatic phototrophic and heterotrophic eukaryotes and biocompatible agents. Adv. Colloid Interface. 169, 59-79 (2011)

20. Lukman, A.I., Gong, B., Marjo, C.E., Roessner, U., Harris, A.T.: Facile synthesis, stabilization and anti-bacterial performance of discrete Ag nanoparticles using medicago sativa seed exudates. J. Colloid Interface. Sci. 353, 433-444 (2011)

21. Kreibig, U., Volmer, M.: Optical Properties of Metal Clusters. Springer, Berlin (1995)

22. Baia, L., Muresan, D., Baia, M., Popp, J., Simon, S.: Structural properties of silver nanoclusters-phosphate glass composites. Vib. Spectrosc. 43, 313-318 (2007)

23. Smitha, S., Nissamudeen, K., Philip, D., Chopchandran, K.: Studies on surface plasmon resonance and photoluminescence of silver nanoparticles. Spectrochim. Acta A 71, 186-190 (2008) 
24. Rodriguez, A.G., de Torres, A.G., Pavon, J.C., Ojeda, C.B.: Simultaneous determination of iron, nickel and cobalt by UVvisible spectrophotometry with multivariate calibration. Talanta 47, 463-470 (1998)

25. Patnaik, P.: Dean's Analytical Chemistry Handbook. McGraw Hill, New York (2004)

26. Kasolo, J.N., Bimenya, G.S., Ojok, L., Ochieng, J., OgwalOkeng, J.W.: Phytochemicals and uses of Moringa oleifera leaves in Ugandan rural communities. J. Med. Plants Res. 4, 753-757 (2010)

27. Ramesh, S., Cohen, Y., Aubach, D., Gedanken, A.: Atomic force microscopy investigation of the surface topography and adhesion of nickel nanoparticles to submicrospherical silica. Chem. Phys. Lett. 287, 461-467 (1998)

28. Hou, Y., Kondoh, H., Ohta, T., Gao, S.: Size-controlled synthesis of nickel nanoparticles. Appl. Surf. Sci. 241, 218-222 (2005)

29. Lin, K.-C., Lin, Y.-C., Chen, S.-M.: A highly sensitive nonenzymatic glucose sensor based on multi-walled carbon nanotube with nickel and copper nanoparticles. Electrochim. Acta 96, 164-172 (2013)

30. Davies, T.J., Compton, R.G.: The cyclic and linear sweep voltammetry of regular and random arrays of microdisc electrodes: theory. J. Electroanal. Chem. 585, 63-82 (2005)
31. Davies, T.J., Banks, C.E., Compton, R.G.: Voltammetry at spatially heterogeneous electrodes. J. Solid State Electrochem. 9, 797-808 (2005)

32. Mamuru, S.A., Ozoemena, K.I.: Heterogeneous electron transfer and oxygen reduction reaction at nanostructured iron(II)phthalocyanine and its MWCNTs nanocomposites. Electroanalytical 22, 985-994 (2010)

33. Mamuru, S.A., Ozoemena, K.I., Fukuda, T., Kobayashi, N.: Iro$\mathrm{n}$ (II) tetrakis (diaquaplatinum) octacarboxyphthalocyanine supported on multi-walled carbon nanotubes platform: an efficient functional material for enhancing electron transfer kinetics and electrocatalytic oxidation of formic acid. J. Mater. Chem. 20, 10705-10715 (2010)

34. Mamuru, S.A., Ozoemena, K.I.: Impedimetric and electrocatalytic properties of nanostructured iron(II) phthalocyanine at pyrolytic graphite electrode. Mater. Chem. Phys. 114, 113-119 (2009)

35. Orazem, M., Tribollet, B.: Electrochemical Impedance Spectroscopy. Wiley, Hoboken, NJ (2008)

36. Laviron, E.: General expression of the linear potential sweep voltammogram in the case of diffusion less electrochemical systems. J. Electroanal. Chem. 101, 19-28 (1979) 